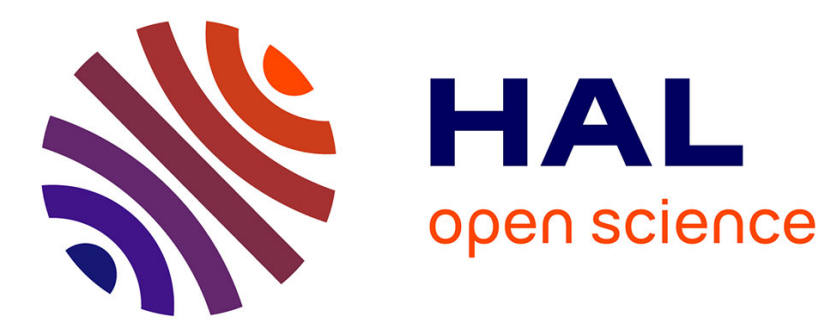

\title{
Metronomic Reloaded: Theoretical Models Bringing Chemotherapy into the Era of Precision Medicine
}

Sébastien Benzekry, Eddy Pasquier, Dominique Barbolosi, Bruno Lacarelle, Fabrice Barlési, Nicolas André, Joseph Ciccolini

\section{- To cite this version:}

Sébastien Benzekry, Eddy Pasquier, Dominique Barbolosi, Bruno Lacarelle, Fabrice Barlési, et al.. Metronomic Reloaded: Theoretical Models Bringing Chemotherapy into the Era of Precision Medicine. Seminars in Cancer Biology, 2015, pp.23. 10.1016/j.semcancer.2015.09.002 . hal-01195547

\section{HAL Id: hal-01195547 https://hal.inria.fr/hal-01195547}

Submitted on 8 Sep 2015

HAL is a multi-disciplinary open access archive for the deposit and dissemination of scientific research documents, whether they are published or not. The documents may come from teaching and research institutions in France or abroad, or from public or private research centers.
L'archive ouverte pluridisciplinaire HAL, est destinée au dépôt et à la diffusion de documents scientifiques de niveau recherche, publiés ou non, émanant des établissements d'enseignement et de recherche français ou étrangers, des laboratoires publics ou privés. 


\section{METRONOMIC RELOADED: THEORETICAL MODELS BRINGING CHEMOTHERAPY INTO THE ERA OF PRECISION MEDICINE}

Sébastien Benzekry ${ }^{1}$, Eddy Pasquier ${ }^{2,3,4}$, Dominique Barbolosi ${ }^{3}$, Bruno Lacarelle ${ }^{3,5}$, Fabrice Barlési ${ }^{3,6}$, Nicolas André, , $^{2,7}$ and Joseph Ciccolini ${ }^{3,5}$

1. INRIA, team MONC, Institut de mathématiques de Bordeaux UMR 5251, Université de Bordeaux, Talence

2. Metronomics Global Health Initiative, Marseille, France

3. INSERM UMR S_911 CRO2, Aix Marseille Univ, Marseille, France

4. Children's Cancer Institute, Randwick, Australia

5. Clinical Pharmacokinetics La Timone University Hospital of Marseille, Marseille France.

6. Service d'Oncologie Multidisciplinaire et d'Innovations Thérapeutiques, Nord University Hospital of Marseille, Marseille France.

7. Service d'Hematologie et Oncologie Pediatrique, La Timone Hospital, Marseille, France

\section{Correspondence to:}

Dr Joseph Ciccolini, SMARTc, Pharmacokinetics Unit, Faculté de Pharmacie de Marseille, 27 Bd Jean Moulmin 13385 Marseille 05 France. Email :

joseph.ciccolininiv-amu.fr

\section{Keywords}

Metronomic chemotherapy, mathematical modeling, $\mathrm{PK} / \mathrm{PD}$, precision medicine 


\begin{abstract}
Oncology has benefited from an increasingly growing number of groundbreaking innovations over the last decade. Targeted therapies, biotherapies, and the most recent immunotherapies all contribute to increase the number of therapeutic options for cancer patients. Consequently, substantial improvements in clinical outcomes for some disease with dismal prognosis such as lung carcinoma or melanoma have been achieved. Of note, the latest innovations in targeted therapies or biotherapies do not preclude the use of standard cytotoxic agents, mostly used in combination. Importantly, and despite the rise of bioguided (a.k.a. precision) medicine, the administration of chemotherapeutic agents still relies on the maximum tolerated drug (MTD) paradigm, a concept inherited from theories conceptualized nearly half a century ago. Alternative dosing schedules such as metronomic regimens, based upon the repeated and regular administration of low doses of chemotherapeutic drugs, have emerged as possible strategies to improve response rates while reducing toxicities. The recent changes in paradigm in the way we theorize cancer biology and evolution, metastatic spreading and tumor ecology, alongside the recent advances in the field of immunotherapy, have considerably strengthened the interest for metronomic approaches. This paper aims at reviewing the recent evolutions in the field of theoretical biology of cancer and computational oncology, with a focus on the consequences these changes have on the way we administer chemotherapy. In particular, a step towards developing adaptive dosing should help to further optimize the efficacy of metronomic therapy. There is a rising trend to establish personalized medicine in oncology. Developing extensive bioguided strategies for decision-making in the choice of drugs to be administered is now a common practice at the bedside. Similarly, developing extensive model-guided strategies for decision-making in refining dosing and scheduling should be undertaken to achieve precision medicine in oncology.
\end{abstract}

\title{
INTRODUCTION
}


Innovative technologies have dramatically changed the way we treat cancer. From crude surgery for centuries, to the introduction of radiotherapy in the 1930's and that of chemotherapy in the 1950's [1], we can now envision the development of personalized treatments for cancer patients, thanks to the advances made in biology, chemistry, physics, mathematics and engineering. Immune checkpoint inhibitors, antiangiogenics and targeted therapies have already entered the clinic with various level of success, and innovative technologies in imaging, PK/PD modeling and the omics are helping clinicians in their decision making on a daily basis. Yet, chemotherapy is still administered today almost the exact same way it was fifty years ago. Why is that?

Here we will try to address this question by looking back at the early theoretical concepts that led to the development and widespread use of what is now called "conventional chemotherapy" - i.e. the administration of chemotherapy at or close to the maximum tolerated dose (MTD), with drug-free breaks in between cures to allow for the patient to recover from the treatment-related toxicities. We will explain the limitations of these early theoretical and mathematical models and discuss ways they can be improved to better take into account the complexity and rapid evolution of tumors and ultimately optimize treatment efficacy. This will lead us to present innovative theoretical models that support alternative ways of administering chemotherapy. These include metronomic chemotherapy [2-4] - i.e. the frequent administration of chemotherapeutic drugs at relatively low, non-toxic doses, without prolonged drug-free break - and adaptive therapy $[5,6]$ - i.e. modulating the dose and frequency of chemotherapy administrations in order to maintain a constant tumor volume. Metronomic chemotherapy is expected to bring substantial benefit over existing MTD regimen by interfering with novel targets at the tumor level or the tumor micro-environment level (Figure 1). In many respects, developing metronomic chemotherapy could pave the way for implementing computational oncology at bedside, because optimizing metronomic regimen should only be achieved thanks to modeling support. 


\section{Historical concepts}

Fifty years ago, Skipper, Schabel and Wilcox were the first to introduce theoretical concepts for the optimal design of chemotherapy schedules [7]. Based on experimental studies involving L1210 leukemic cells — which exhibit exponential growth when left untreated - , they introduced and demonstrated the log-kill effect for several cytotoxic agents, including 6-mercaptopurine, 5-fluoruracil and vinblastine [7]. This principle, based on an analogy with the law of mass action for kinetic reactions in chemistry, states that exposure to a given amount of drug kills a constant fraction of a cancer cell population, hence reducing it of a constant amount in logarithmic scale (Figure 2). For instance, if a drug has a one log-kill effect, then it will reduce a population of $10^{6}$ cells to $10^{5}$ cells and a population of $10^{2}$ cells to $10^{1}$ cells. Further on, based on their experimental work that demonstrated that the presence of as little as one single leukemic cell was sufficient to lead to the host death, they argued that the goal of the therapy should be to achieve complete cure of the disease, i.e. eradication of all malignant cells. In this context, they demonstrated that a large-dose/short time (single administration) schedule was superior to a chronic (daily) low-dose schedule (with similar or larger total dose) [8]. However, when this view (that was involved in the calculation of the number of cycles required for cure) was applied to the adjuvant systemic treatment of micrometastases (for breast cancer for instance), it did not lead to the expected results [9]. Two major criticisms were addressed to the work of Skipper et al.: 1) they considered a homogeneously sensitive population of cancer cells (i.e. no resistance was explicitly taken into account) and 2) the experimental system they employed was limited to a single leukemic cell line and their conclusions might not extend to solid tumors.

Regarding point 1), substantial efforts in the modeling of resistance to cytotoxic agents have been provided by the work of Goldie and Coldman [10]. The Goldie-Coldman model states that mutation rates towards resistance are relatively high within a population of tumor cells and that mutations develop spontaneously during the natural course of the disease (innate resistance). This implies that the treatment should start as soon as possible in order to avoid the natural tumor progression leading to the presence 
of more resistant cells. It also implies that if several drugs are to be administered and cannot be given simultaneously for toxicity constraints, they should be delivered alternatively in order to avoid resistance to drug B to develop during therapy with drug A. However, predictions of this model were ruled out by several trials, in breast cancer patients for instance, where strategies that delay therapy or did not respect strict alternation of combination regimen were proven to perform at least as well as the Goldie-Coldman recommended strategy [11]. One of the main hypotheses underlying the Goldie-Coldman model is the concept of absolute resistance, which is discussable. Indeed, there is some evidence that tumors can exhibit various levels of relative drug resistance [12].

In the 1960's, thorough study of tumor kinetics led to the realization that the specific tumor growth rate for solid tumors, instead of being constant (exponential growth), was rather a decreasing function of the volume, provided tumor growth is observed long enough [13-15]. In mathematical terms, tumor growth can be formalized by means of the following differential equation

$$
\frac{d V}{d t}=f(V) V
$$

which equates the (infinitesimal) increase in tumor volume (size) per unit of time on the left hand side and a function of the volume on the right hand side (the growth rate). In the expression above, function $f$ is the specific growth rate that was evidenced as being decreasing, first in animal studies by growth curve analysis [16,17] followed by clinical investigations. The latter were either directly based on patient data such as direct cells counts in IgG multiple myeloma patients [18] or mammographic measurements of breast tumors [13]; or indirectly from survival data of untreated patients [19] or the distribution of tumor size in the patient population [20]. More specifically, investigators demonstrated that the growth curve patterns could be well described by several structural expressions, the most famous (and employed) being the Gompertz law $(f(V)=\alpha-\beta \ln (V))) \quad[16,19,21-23]$. This expression is not the only one able to describe the empirical data and other models such as the power law $(f(V)=$ 
$a V^{y-1}$ ) can do as good as the Gompertz, while their etiology might be better grounded (fractional dimensional of the vasculature) [17,20,23,24]. We refer to [23] for a recent review of the descriptive and predictive properties of these models for experimental data.

In the 1970's, L. Norton and R. Simon revisited the Skipper-Schabel-Wilcox log-kill hypothesis, in order to extend it to growing tumors with non-constant specific growth rate [25]. They suggested that since chemotherapy is mostly based on anti-mitotic agents, it should only be active against these cells that are actively proliferating, i.e. precisely the ones that contribute to the volume increase in equation (1) [25]. Moreover, the current view of the effect of chemotherapy (log-kill) was in contradiction with clinical observations such as: a) the effect on small tumors in an adjuvant setting was not as pronounced as expected and b) decreased sensitivity to therapy was observed also for very large tumors. Instead of a killing term proportional to the volume of the tumor, they proposed a killing term proportional to the tumor growth rate, i.e.:

$$
\frac{d V}{d t}=f(V) V(1-C(t))
$$

They demonstrated that their model was consistent with clinical observations, able to fit preclinical experiments and was superior to the log-kill model in predicting the future course of an experimental treated growth curve from a few initial measurements [25]. This model has profound clinical implications. First, it predicts a superiority of densified dosing regimens. Indeed, if less inter-cycle time is allowed to the tumor to regrow, it reaches a smaller size at the beginning of the next cycle, thus a larger growth rate (Gompertzian growth) and consequently a larger amount of cells killed by the drug [25]. This prediction was confirmed by clinical trials that densified administration drug schedules from every 21 days to every 14 days and showed benefit of applying the second regimen [26]. Second, extending these concepts to drug combinations, the Norton-Simon model advocates for sequential administration of the drugs (in order to densify the treatment for each drug separately), as opposed to the 
strict alternation supported by the Goldie-Coldman model. Clinical trials (for the treatment of breast cancer for instance) again confirmed this prediction of the NortonSimon model [27,28]. This example demonstrates how rational thinking and mathematical methods, based on phenomenological theories, can help to successfully guide clinical trials $[29,30]$. Under the Norton-Simon hypothesis, larger absolute kill of cancer cells resulting from early chemotherapy, as opposed to late chemotherapy, might be counter-balanced by faster regrowth due to high growth rates of small tumors (see Figure 2, right panel), thus leading to similar overall survival in both cases, despite significantly different times to relapse [31].

\section{Modern cancer biology}

During the early phases of the development of chemotherapy, the ultimate goal of therapy was always complete cure from the disease. As stated by Skipper et al.: “(...) it appears that high-level, short-term schedules offer considerably greater potential for obtaining "cures"" [7]. Interestingly, even if these investigators were pleading for this option, they already had noticed that this might not be the best strategy to achieve best long-term control of the disease. Indeed, the previous quote continues with "This preference does not necessarily hold with regards to achieving maximum increase in life span of animals which die in spite of therapy". Since several years, there is an increasing trend towards a paradigm shift in clinical oncology: in view of the failure to cure patients using conventional approaches, investigators proposed to change the goal of therapy from complete eradication of the tumor to a long-term management of the disease [6]. This suggests that instead of waiting for "magic bullets" that would provide an absolute and complete solution, one might instead look into optimization of already existing therapeutic tools. In order to improve current cancer treatments, historical concepts need to be revised to better take into account the complexity of the disease, including the impact of the tumor microenvironment (e.g. vasculature and immune system) and clonal heterogeneity on the efficacy of chemotherapy protocols. 


\section{Tumor heterogeneity and (epi)-genetic resistances}

In a very elegant, simple and conceptually powerful theoretical study, Hahnfeldt, Folkman and Hlatky re-analyzed the problem of dose repartition in light of tumor heterogeneity [32]. They considered two subpopulations of cancer cells with two distinct sensitivities to a given drug, with transition rates between the two. Importantly, the transition rate from the less sensitive population to the most sensitive one was assumed to be positive, thus allowing for a resensitization effect. Under their minimal framework that allows to perform explicit computations, they demonstrated that more regularly spaced dosing of the drug yields better final tumor reduction when compared to irregular spacing, for both single and multiple cycles therapies. Eventually, based on the assumption that the endothelium is more subject to resensitization than the tumor compartment, they also proposed their model as an explanation for the anti-angiogenic basis of metronomic chemotherapy [33]. Along the same lines (i.e. heterogeneous population of cancer cells composed of both resistant and sensitive cells that can switch phenotypes), and adding stochasticity in the dynamics (Markov Chain process), Liao et al. recently developed a graphical tool, termed metronogram, which provides indications on how to adapt the period between subsequent administrations of the drug in order to maintain anti-tumor efficacy [34].

Inspired by the example of invasive species in ecology and the use of pesticides, a situation where complete eradication is usually impossible due to high phenotypic diversity that leaves resistant individuals after intervention, R. Gatenby proposes to see a tumor as an ecosystem ruled by evolutionary laws [6]. In this context, high dose chemotherapy might have the deleterious effect of selecting for the most resistant cells, by eliminating the sensitive competitors. On the other hand, a low-dose continuous therapy, by keeping a positive amount of sensitive cells, might provide a better longterm control of the total population [6]. In a study using both mathematical and experimental approaches, Gatenby and colleagues further developed this concept and introduced the idea of adaptive therapy. It consists in modulating the dose and frequency of therapeutic administrations in order to maintain a constant tumor volume, as opposed to the conventional approach that administers a fixed dosing regimen 
repeated over several cycles [5]. Although performed on only one (ovarian) cancer cell line and resulting in a (possibly toxic) larger total dose, the adaptive schedule was found to be superior to the standard MTD one, yielding excellent long-term management of the tumor mass.

Applying the same evolutionary concepts to population dynamics of cancer cells, Clairambault et al. developed a modeling framework that revisited the concepts underlying the Delbrück-Luria/Goldie-Coldman model of spontaneous development of resistance [35]. In their work, they made two major hypotheses: 1) reversible, druginduced (rather than irreversible and spontaneous) development of resistance, based on a recent experimental study and 2) varying levels of resistance (instead of the classic sensitive/resistant dichotomy). Point 2) was elegantly formalized in mathematical terms within the context of structured evolutionary population dynamics, by means of one (or more) continuous variable representing a phenotyping trait [36,37]. Full implications of these recent studies in terms of optimal scheduling have not yet been published but will probably generate non-trivial insights on optimal administration schedules emerging from the complex dynamics due to tumor heterogeneity.

\section{Anti-angiogenesis}

Metronomic chemotherapy was initially developed to potentiate the anti-angiogenic properties of chemotherapeutic drugs [33,38]. This target switch has important implications in terms of optimal scheduling for the delivery of a cytotoxic agent. In 1999, Hahnfeldt et al. derived a biologically-based (yet technically simple) mathematical model for tumor growth under endogenous angiogenic signaling that was able to describe the effect of several anti-angiogenic molecules [39]. Based on this model, d'Onofrio and Gandolfi studied the effect of scheduling variations and obtained that a drug targeting the vasculature would have a better effect if administered more frequently at lower doses (assuming constant total dose) [40]. When the drug effect was further assumed to depend on the vascular density, a nontrivial optimal metronomic inter-administration time was found [41]. Interestingly, metronomic scheduling additionally exhibited enhanced robustness towards noise-induced transitions (i.e. escape from therapeutic control) in response to stochasticity in the 
clearance rate [42]. Going further in the implications of the Hahnfeldt model, Ledzewicz and Schättler performed a full optimal control analysis for theoretical optimization of the scheduling of a drug with anti-angiogenic properties [43]. Interestingly, they found that the optimal control, instead of having a bang-bang expression (constant full-dose sections separated by breaks - i.e. conventional chemotherapy), could exhibit singular arc portions where the drug is administered at a lower dose than the MTD, with varying rates. This is the expression in mathematical terms of the concept of a biologically optimal dose, which might differ from the MTD. Recently, mathematical models specifically tailored for the analysis of the antiangiogenic effect of metronomic chemotherapy in various concrete clinical settings were introduced [44-46]. All these rely on the same assumptions: a) chemotherapy has an anti-angiogenic effect by killing endothelial cells in addition to its cytotoxic effect on cancer cells $[38,47]$, b) cancer cells develop resistance whereas endothelial cells do not (due to larger genetic stability) and c) drug action is stronger on endothelial cells than on tumor cells [47]. Using realistic pharmacokinetic models and an interface model for description of the efficacy from the concentration of the drug in the central compartment, MTD and metronomic schedules were compared in silico for the administration of docetaxel [44] or etoposide [45]. In the docetaxel study, the effect on metastatic dissemination was also taken into account. Interestingly, the simulations revealed a long-term beneficial effect of the metronomic regimens, despite an initial superiority of the MTD schedule (Figure 3). Indeed, examination of the dynamic properties of the system revealed that, under the MTD schedule, the fast endothelial dynamics generated sharp relapses during the drug-free breaks that ultimately led to global relapse due to resistance (Figure 3). On the other hand, the metronomic regimen ensured a more stable action on the endothelium that ultimately led to oxygen and nutrient deprivation of the tumor. Of note, when the daily dose of the metronomic schedule was too low (e.g. $8 \mathrm{mg} /$ day), the treatment could not suppress the growth of the tumor, which escaped therapy. This result emphasizes the existence of a minimal dosing schedule (minimally effective dose) that could be computed using the mathematical model, provided it is properly calibrated on patient data. This dose 
depends on specific model parameters that might vary among patients. It is important to note that the determination of biologically optimal dose requires reliable biomarkers. Several markers of response to metronomic chemotherapy have been suggested over the years, such as plasma levels of endogenous angiogenesis inhibitor thrombospondin-1 and the number and viability of circulating endothelial cells [48]. However, correlations between these biomarkers and study endpoints were rare and variable, making them unsuitable for model parameterization.

Similar mathematical model for anti-tumor efficacy can be complemented with a model for hematologic toxicity (i.e., neutrophil counts), thus opening the way to developing more sophisticated constraint-models able to calculate optimal dosing for maximal efficacy while respecting clinical requirements on a certain level of acceptable hematological toxicities [49].

In the clinic, most cancer-related deaths are not due to the primary tumor, but rather to the metastases [50]. To address the issue of treatment on a population of tumors (rather than a single tumor), as well as the effect on the dissemination process, Benzekry and Hahnfeldt [51] defined an optimal control problem written for an organism-scale model of metastatic development. Although complete mathematical analysis was too complicated to be achieved, they could perform a simulation study and found that the overall efficacy of cytotoxic agents, either alone or in combination with antiangiogenic drugs, was generally maximized when employing a metronomic schedule rather than the MTD. Interestingly, in some instances (e.g. values of the parameters, objective function considered), differences in the best strategy occurred between the treatment of the (isolated) primary tumor and the treatment of the (systemic) cancer at the organism scale. These theoretical predictions were independently confirmed in recent pre-clinical studies using mouse models of spontaneous metastases [52].

\section{Role of the immune system}

The role of the immune system in cancer biology and response to treatment is becoming increasingly recognized and has major therapeutic implications [53]. The genetic and cellular alterations that define cancer provide the immune system with the means to generate $\mathrm{T}$ cell responses that recognize and eradicate cancer cells. However, 
elimination of cancer by $\mathrm{T}$ cells has to be balanced with the recognition of non-self and the prevention of auto-immunity. Identification of $\mathrm{T}$ cell inhibitory signals induced by cancer cells, including PD-L1, has led to the development of a new class of cancer immunotherapy that specifically hinders immune effector cell inhibition, reinvigorating and potentially expanding preexisting anticancer immune responses. The presence of suppressive factors in the tumor microenvironment may explain the limited activity observed with previous immune-based therapies and why these therapies may be more effective in combination with agents that target other steps of the cycle. Recent clinical data confirm that cancer immunotherapy is likely to become a key part of the clinical management of cancer and numerous innovative drugs are yet to come [54]. Elsewhere, several pre-clinical and clinical studies have shown that changing the administration schedule from MTD to a metronomic protocol considerably impacts the effects of chemotherapy on the immune system, switching from immunosuppression to immunostimulation [3].

Recently, Ledzewicz and Schättler proposed a model integrating three components for the effect of chemotherapy: a direct cytotoxic effect on cancer cells, an anti-angiogenic effect and an immune-stimulatory effect [55]. They mathematically investigated the dynamics of the resulting system under constant infusion therapy. However no study of the effect of the scheduling has yet been performed in this model or validated in vivo. Using ectotopic glioma mouse models, Doloff and Waxman demonstrated that a metronomic cyclophosphamide regimen, rather than exerting anti-angiogenic effects, elicited anti-tumor innate immunity, including recruitment of macrophages, dendritic and NK cells [56]. Going further, the same group then reported a schedule dependence of this anti-tumor innate immune effect of metronomic chemotherapy, with significant differences in growth curves for cyclophosphamide administered either every 3 days or every six days (the classical metronomic regimen [38]), while total exposure was kept constant [57]. Their results highlight the potentiality of model-driven optimization of the scheduling in order to find non-trivial optima for dose and frequency of administration. 
In a theoretical study, I. Kareva proposed a model for interactions between cancer stem cells, non-stem cancer cells, and cytotoxic lymphocytes [58]. By suggesting that the non-stem cancer cells might provide a protective shield to the cancer stem cells, she developed a mechanistic rationale for the use of metronomic chemotherapy: frequent administration of a cytotoxic drug at low dose might provide a continuous pealing of the non-stem cancer cells layers protecting the cancer stem cells, while not depleting too strongly the lymphocyte population, therefore restoring anti- cancer stem cells immunity. Of note, the immunomodulatory properties of metronomic chemotherapy may vary according to the cytotoxics used [59]. Regulatory $\mathrm{T}$ cells for instance may be targeted using metronomic temozolomide or cyclophosphamide, myeloid-derived suppressor cells can be modulated using metronomic gemcitabine and 5-fluorouracil, innate immune response can be activated by metronomic temozolomide while maturation of dendritic cells can be induced by metronomic vinblastine [4]. Future computational models will need to take into account these specificities in order to develop optimal metronomic protocols.

\section{Era of Metronomic 2.0. : are we there yet?}

Deciphering the mechanisms responsible for the antitumor activity of metronomic regimens is particularly challenging because of the overlap between anti-angiogenic, immune-stimulatory and cytotoxic effects. Importantly, each drug has its own mechanism of action, multiple drugs can be combined, and each drug can be given following a near infinite number of combinations depending on dose, frequency and duration of administration. As a consequence, standard empirical designs to test metronomic regimens are unlikely to identify the optimal combination between drugs, dosing and scheduling, to achieve the best efficacy in cancer patients. In this respect, mathematical modeling can be regarded as a critical and powerful new tool in decision-making, provided that suitable models are made available and validated. Such models will allow testing numerous hypotheses in silico so as to identify, in a cost-, time- and ethically-effective manner, the best regimen among countless possibilities. 
Implementation of computational oncology into metronomic can be achieved using mechanistic models. Those models take into account all the components related to tumor biology, plus the intrinsic mechanisms of action of the drugs, thus leading to a multi-scale model encapsulating all the items to be taken into account to achieve some antitumor efficacy eventually. Another strategy is to develop phenomenological models, aiming at describing, with no or little mechanistic basis, the impact of metronomic regimen, mostly on a simplified PK/PD basis, with some critical features such as integration of a resistance component. Such a model has been recently proposed by Faivre et al. for the administration of temozolomide [45], and used in silico as a tool to compare metronomic and MTD administrations, thus predicting the respective efficacy of these regimens. The same model has been adapted and extended to oral vinorelbine, another cytotoxic drug frequently administered following metronomic schedules [46]. In this study, the model was used to identify an optimal metronomic protocol for lung cancer patients, based upon clinical data already published in patients with solid tumors. In silico simulations suggested that an alternative 60-30-60 mg dosing given on a D1-D2-D4 basis should lead to higher efficacy with a good tolerance as compared with all other metronomic regimens tested thus far. Of note, such contra-intuitive schedule could not have been identified simply by analyzing the results of the dozen of phase I or phase II trials conducted with metronomic vinorelbine. Finally, this kind of simplified, phenomenological model was used to drive metronomic gemcitabine in a non-clinical study [60]. Data from tumorbearing mice showed outstanding response in a resistant model of neuroblastoma, following a metronomic scheduling (i.e., $1 \mathrm{mg} / \mathrm{kg} / \mathrm{d}$ over 28 days) identified by a mathematical model among a variety of possible combinations between doses, frequency and duration [61,62]. This result highlights how model-driven metronomic can improve the efficacy of chemotherapy indeed. The converging advances in the fields of mathematics, tumor biology and pharmacology, advocate for the development of modeling supports (either mechanistic or merely descriptive). In particular, beyond the very concept of metronomic administration, refining dosing and scheduling through adaptive strategies is a challenging task requiring specific modeling resources. 
For instance, inter-patient variability affecting pharmacodynamic determinants (i.e. target expression or target mutation, genetic or molecular dysregulations on downstream signaling pathways) or pharmacokinetic determinants (i.e., drug metabolizing enzymes, membrane transporters, upstream transcription factors regulating both enzymes and transporters expression) can be major causes for treatment failure upon standard dosing [63]. In this respect, developing global tools encompassing PK/PD models for guiding metronomic administration, plus possible pharmacogenomic and pharmacogenetic determinants to be used as covariates for further tailor dosing, should help to improve clinical outcome. Only a few studies have presented such model-driven dosing strategies that take into account individual factors of variability such as genetic polymorphisms affecting PK/PD relationships. Yet, appealing results in terms of prediction or improvement of the clinical outcome in patients with cancer treated with standard dosing have been reported [64, 65]. In addition to tailored dosing, modeling pharmacogenomic determinant should help to forecast treatment efficacy, thus potentially helping the decision-making to treat, or not, certain patients. For instance, genetic profiling of circulating tumor and invasive cells in patients with pancreatic cancer and development of a dedicated model, allowed predicting effective and ineffective chemotherapeutic agents. In this blind study, clinical outcome proved to match initial model predictions, thus suggesting that a prospective use of the model for decision-making could have avoided ineffective treatments [66]. To what extent genetic polymorphisms could be considered as biomarkers when performing metronomic administration remain to be fully elucidated before being implemented in dedicated models. For instance, using low doses of cytotoxics is usually less likely to saturate enzymatic systems or active transporters, thus smothering the impact of inter-patient variability in terms of decreased expression or activity in drug transport or disposition in the body [67]. Conversely, ultra-metabolizer patients could become more rapidly prone to treatment failure by an increased liver clearance [68]. Further prospective studies are warranted to test all these model-based strategies with metronomic approaches, since inter-patient variability is a challenging issue that remains largely underestimated. 
In this context, metronomic illustrates how the time for trial and error practices is now over, and calls for developing computationally-driven regimens as a means to optimize anticancer therapies in the $21^{\text {st }}$ century.

\section{Conclusion}

Modern cancer biology has accumulated a large amount of knowledge about the various players involved in neoplasia and their interactions. Consequently, it becomes more and more difficult to determine the outcome of therapeutic intervention using cytotoxic agents (in monotherapy or in combination with other drugs) using rational thinking alone. In this regard, mathematical and computational models offer powerful tools that are currently under-utilized but could help in: 1) conceptualizing our theoretical thinking of the disease and the dynamics of therapeutic interventions, 2) identifying critical variables and parameters, 3) potentiating the analysis of available data and/or patient biomarkers and 4) simulating the outcome of complex situations. Under these lines of reasoning they could offer invaluable help to: 1) test a wide range of possibilities in silico for the design of anti-cancer drug regimens before validation of these innovative protocols in animal models and 2) personalize the dosing of anti-cancer agents, using patient-specific model parameters informed by biomarkers.

Future efforts are needed both in terms of precisely defining the essential dynamics to be modeled for a given pathology, clarifying the objectives to be optimized, and identifying reliable biomarkers and their connections to model parameters. 


\section{FIGURES LEGENDS}

Figure1: Metronomic VS. MTD chemotherapy. Alternative dosing and scheduling is expected to achieve higher antiproliferative efficacy via novel mechanisms of action such as anti-angiogenic effect, action on cancer stem cells or immuno-stimulating properties, in addition to direct cytotoxicity on tumor cells.

Figure 2: Left: Skipper-Schabel-Wilcox log-kill model. Tumor growth is exponential (linear in log-scale) and each cycle of chemotherapy results in removal of a constant fraction of the tumor volume (as opposed to a constant amount of cells). This is reflected by a constant log-kill. The simulation assumes a log-kill of three (i.e. reduction of $99.9 \%$ of the tumor mass) over six three-weeks cycles, for an initial total tumor load of $10^{9}$ cells. The dashed line represents the size of one cell, that classical MTD chemotherapy approaches consider as the goal to achieve for eradication of the disease. Right: Norton-Simon model. Untreated tumor growth is Gompertzian and exhibits a decreasing specific growth rate. The Norton-Simon hypothesis implies a larger log-kill for smaller tumors and suggests to densify the chemotherapy administration protocol. This is illustrated by comparison of a three-weeks regimen (black curve) and a densified two-weeks regimen (grey curve). The latter exhibits deeper drop of the tumor burden and thus larger probability of "cure". However, note that when tumor regrows, both schedules have the same time to recurrence.

Figure 3: MTD vs Metronomic. Simulations of a mathematical model for the antiangiogenic effect of metronomic therapy for Docetaxel [44]. The MTD schedule is defined by $100 \mathrm{mg} / \mathrm{m}^{2}$ on the first day of a 21-days cycle. The metronomic schedule is defined by $10 \mathrm{mg} / \mathrm{m}^{2}$ daily with no drug-free breaks. Left: tumor volume. Right: carrying capacity, assumed to represent the vasculature. Despite initial superiority of the MTD regimen during the first cycles, on the long run the metronomic regimen achieves better tumor control. This is due to the intrinsically fast dynamics of the 
endothelium that recovers during drug free intervals with the MTD schedule (right panel). 


\section{References}

[1] Mukherjee S. The Emperor of All Maladies: A Biography of Cancer. Reprint edition. New York: Scribner; 2011.

[2] Hanahan D, Bergers G, Bergsland E. Less is more, regularly: metronomic dosing of cytotoxic drugs can target tumor angiogenesis in mice. J Clin Invest 2000;105:1045-7.

[3] Pasquier E, Kavallaris M, André N. Metronomic chemotherapy: new rationale for new directions. Nat Rev Clin Oncol 2010;7:455-65.

[4] André N, Carré M, Pasquier E. Metronomics: towards personalized chemotherapy? Nat Rev Clin Oncol 2014;11:413-31.

doi:10.1038/nrclinonc.2014.89.

[5] Gatenby RA, Silva AS, Gillies RJ, Frieden BR. Adaptive Therapy. Cancer Res 2009;69:4894-903. doi:10.1158/0008-5472.CAN-08-3658.

[6] Gatenby RA. A change of strategy in the war on cancer. Nature 2009;459:508-9. doi:10.1038/459508a.

[7] Skipper HE, SCHABEL FM, WILCOX WS. Experimental evaluation of potential anticancer agents XIII. On the criteria and kinetics associated with "curability" of experimental leukemia. Cancer Chemother Rep 1964;35:1-111.

[8] Skipper HE. The effects of chemotherapy on the kinetics of leukemic cell behavior. Cancer Res 1965;25:1544-50.

[9] Early Breast Cancer Trialists' Collaborative Group (EBCTCG). Systemic treatment of early breast cancer by hormonal, cytotoxic, or immune therapy. 133 randomised trials involving 31,000 recurrences and 24,000 deaths among 75,000 women. Lancet 1992;339:1-15.

[10] Goldie JH, Coldman a J. A mathematic model for relating the drug sensitivity of tumors to their spontaneous mutation rate. Cancer Treat Rep 1979;63:1727-33.

[11] Frei E, Freireich EJ, Gehan E, Pinkel D, Holland JF, Selawry O, et al. Studies of Sequential and Combination Antimetabolite Therapy in Acute Leukemia: 6Mercaptopurine and Methotrexate. Blood 1961;18:431-54.

[12] Kern MA, Helmbach H, Artuc M, Karmann D, Jurgovsky K, Schadendorf D. Human melanoma cell lines selected in vitro displaying various levels of drug resistance against cisplatin, fotemustine, vindesine or etoposide: modulation of proto-oncogene expression. Anticancer Res 1997;17:4359-70.

[13] Spratt JA, von Fournier D, Spratt JS, Weber EE. Decelerating growth and human breast cancer. 1993;71:2013-9.

[14] Steel GG, Lamerton LF. The growth rate of human tumours. Br J Cancer 1966;20:74-86.

[15] Wheldon TE. Mathematical models in cancer research. Hilger Bristol; 1988.

[16] Laird AK. Dynamics of tumor growth. Br J Cancer 1964;13:490-502.

[17] Dethlefsen L a, Prewitt JM, Mendelsohn ML. Analysis of tumor growth curves. J Natl Cancer Inst 1968;40:389-405.

[18] Sullivan PW, Salmon SE. Kinetics of tumor growth and regression in IgG multiple myeloma. J Clin Invest 1972;51:1697-708. doi:10.1172/JCI106971.

[19] Norton L. A Gompertzian model of human breast cancer growth. Cancer 
Res 1988;48:7067-71.

[20] Hart D, Shochat E, Agur Z. The growth law of primary breast cancer as inferred from mammography screening trials data. Br J Cancer 1998;78:382-7.

[21] Casey AE. The Experimental Alteration of Malignancy with an Homologous Mammalian Tumor Material : I . Results with Intratesticular Inoculation. Am J Cancer 1934;21:760-75.

[22] Norton L, Simon R, Brereton HD, Bogden AE. Predicting the course of Gompertzian growth. Nature 1976;264:542-4.

[23] Benzekry S, Lamont C, Beheshti A, Tracz A, Ebos JML, Hlatky L, et al. Classical mathematical models for description and prediction of experimental tumor growth. PLoS Comput Biol 2014;10:e1003800.

doi:10.1371/journal.pcbi.1003800.

[24] West GB with Herman AB, Van M Savage. A Quantitative Theory of Solid Tumor Growth, Metabolic Rate and Vascularization. PLoS ONE 2011;6:e22973. doi:10.1371/journal.pone.0022973.

[25] Norton L, Simon R. Tumor size, sensitivity to therapy, and design of treatment schedules. Cancer Treat Rep 1977;61:1307-17.

[26] Held G, Schubert J, Reiser M, Pfreundschuh M. Dose-Intensified Treatment of Advanced-Stage Diffuse Large B-Cell Lymphomas. Semin Hematol 2006;43:221-9. doi:10.1053/j.seminhematol.2006.07.003.

[27] Bonadonna G, Zambetti M, Moliterni A, Gianni L, Valagussa P. Clinical relevance of different sequencing of doxorubicin and cyclophosphamide, methotrexate, and Fluorouracil in operable breast cancer. J Clin Oncol 2004;22:1614-20. doi:10.1200/JC0.2004.07.190.

[28] Citron ML, Berry DA, Cirrincione C, Hudis C, Winer EP, Gradishar WJ, et al. Randomized trial of dose-dense versus conventionally scheduled and sequential versus concurrent combination chemotherapy as postoperative adjuvant treatment of node-positive primary breast cancer: first report of Intergroup Trial C9741/Cancer and Leukemia Group B Trial 9741. J Clin Oncol 2003;21:1431-9. doi:10.1200/JC0.2003.09.081.

[29] Norton L, Simon R. The Norton-Simon hypothesis revisited. Cancer Treat Rep 1986;70:163-9.

[30] Simon R, Norton L. The Norton-Simon hypothesis: designing more effective and less toxic chemotherapeutic regimens. Nat Clin Rev Oncol 2006;3:406-7.

[31] Dang C, Gilewski TA, Surbone A, Norton L. Growth Curve Analysis. Holl.Frei Cancer Med., BC Decker; 2003.

[32] Hahnfeldt P, Folkman J, Hlatky L. Minimizing long-term tumor burden : the logic for metronomic chemotherapeutic dosing and its antiangiogenic basis. J Theor Biol 2003;220:545-54.

[33] Kerbel RS, Kamen BA. The anti-angiogenic basis of metronomic chemotherapy. Nat Rev Cancer 2004;4:423-36.

[34] Liao D, Estévez-Salmerón L, Tlsty TD. Conceptualizing a tool to optimize therapy based on dynamic heterogeneity. Phys Biol 2012;9:065005. doi:10.1088/1478-3975/9/6/065005.

[35] Chisholm RH, Lorenzi T, Lorz A, Larsen AK, de Almeida LN, Escargueil A, et al. Emergence of drug tolerance in cancer cell populations: an evolutionary outcome of selection, nongenetic instability, and stress-induced adaptation. Cancer Res 2015;75:930-9. doi:10.1158/0008-5472.CAN-14-2103. 
[36] Lorz A, Lorenzi T, Hochberg ME, Clairambault J, Perthame B. Populational adaptive evolution, chemotherapeutic resistance and multiple anti-cancer therapies. ESAIM Math Model Numer Anal 2013;47:377-99. doi:10.1051/m2an/2012031.

[37] Lorz A, Lorenzi T, Clairambault J, Escargueil A, Perthame B. Modeling the Effects of Space Structure and Combination Therapies on Phenotypic Heterogeneity and Drug Resistance in Solid Tumors. Bull Math Biol 2014;77:122. doi:10.1007/s11538-014-0046-4.

[38] Browder T, Butterfield CE, Kraling BM, Shi B, Marshall B, O'Reilly MS, et al. Antiangiogenic scheduling of chemotherapy improves efficacy against experimental drug-resistant cancer. Cancer Res 2000;60:1878-86.

[39] Hahnfeldt P, Panigrahy D, Folkman J, Hlatky L. Tumor development under angiogenic signaling: a dynamical theory of tumor growth, treatment response, and postvascular dormancy. Cancer Res 1999;59:4770-5.

[40] D' Onofrio A, Gandolfi A, Rocca A. The dynamics of tumour-vasculature interaction suggests low-dose, time-dense anti-angiogenic schedulings. Cell Prolif 2009;42:317-29.

[41] D' Onofrio A, Gandolfi A. Chemotherapy of vascularised tumours: role of vessel density and the effect of vascular n.d.

[42] D' Onofrio A, Gandolfi A. Resistance to antitumor chemotherapy due to bounded-noise-induced transitions. Phys Rev E 2010;82:061901. doi:10.1103/PhysRevE.82.061901.

[43] Ledzewicz U, Schättler H. Antiangiogenic therapy in cancer treatment as an optimal control problem. SIAM J Control Optim 2007;46:1052-79. doi:10.1137/060665294.

[44] Benzekry S, André N, Benabdallah A, Ciccolini J, Faivre C, Hubert F, et al. Modelling the impact of anticancer agents on metastatic spreading. Math Model Nat Phenom 2012;7:306-36.

[45] Faivre C, Barbolosi D, Pasquier E, André N. A mathematical model for the administration of temozolomide: comparative analysis of conventional and metronomic chemotherapy regimens. Cancer Chemother Pharmacol 2013;71:1013-9. doi:10.1007/s00280-013-2095-z.

[46] Barbolosi D, Ciccolini J, Meille C, Elharrar X, Faivre C, Lacarelle B, et al. Metronomics chemotherapy: time for computational decision support. Cancer Chemother Pharmacol 2014;74:647-52. doi:10.1007/s00280-014-2546-1. [47] Klement G, Baruchel S, Rak J, Man S, Clark K, Hicklin DJ, et al. Continuous low-dose therapy with vinblastine and VEGF receptor- 2 antibody induces sustained tumor regression without overt toxicity. J Clin Invest 2000;105:15-24. [48] Cramarossa G, Lee EK, Sivanathan L, Georgsdottir S, Lien K, Santos KD, et al. A systematic literature analysis of correlative studies in low-dose metronomic chemotherapy trials. Biomark Med 2014;8:893-911. doi:10.2217/bmm.14.14.

[49] Meille C, Iliadis A, Barbolosi D, Frances N, Freyer G. An interface model for dosage adjustment connects hematotoxicity to pharmacokinetics. J

Pharmacokinet Pharmacodyn 2008;35:619-33. doi:10.1007/s10928-008-91064.

[50] Chaffer CL, Weinberg RA. A perspective on cancer cell metastasis. Science 2011;331:1559-64. doi:10.1126/science.1203543.

[51] Benzekry S, Hahnfeldt P. Maximum tolerated dose versus metronomic scheduling in the treatment of metastatic cancers. J Theor Biol 2013;335:235-44. 
doi:10.1016/j.jtbi.2013.06.036.

[52] Ebos JML, Mastri M, Lee CR, Tracz A, Hudson JM, Attwood K, et al.

Neoadjuvant antiangiogenic therapy reveals contrasts in primary and metastatic tumor efficacy. EMBO Mol Med 2014;6:1561-76.

doi:10.15252/emmm.201403989.

[53] Chen DS, Mellman I. Oncology meets immunology: the cancer-immunity cycle. Immunity 2013;39:1-10. doi:10.1016/j.immuni.2013.07.012.

[54] Sharma P, Allison JP. The future of immune checkpoint therapy. Science 2015;348:56-61. doi:10.1126/science.aaa8172.

[55] Schättler H, Ledzewicz U, Amini B. Dynamical properties of a minimally parameterized mathematical model for metronomic chemotherapy. J Math Biol 2015:1-26.

[56] Doloff JC, Waxman DJ. VEGF Receptor Inhibitors Block the Ability of Metronomically Dosed Cyclophosphamide to Activate Innate Immunity-Induced Tumor Regression. Cancer Res 2012;72:1103-15. doi:10.1158/0008-5472.CAN11-3380.

[57] Chen C-S, Doloff JC, Waxman DJ. Intermittent metronomic drug schedule is essential for activating antitumor innate immunity and tumor xenograft regression. Neoplasia N Y N 2014;16:84-96.

[58] Kareva I. Immune evasion through competitive inhibition: The shielding effect of cancer non-stem cells. J Theor Biol 2015;364:40-8. doi:10.1016/j.jtbi.2014.08.035.

[59] Galluzzi L, Senovilla L, Zitvogel L, Kroemer G. The secret ally: immunostimulation by anticancer drugs. Nat Rev Drug Discov 2012;11:215-33. doi:10.1038/nrd3626.

[60] Ciccolini J, Pasquier E, Lombard A, Giacometti S, Faivre C, Fanciullino R, et al. Abstract 4506: Computational-driven metronomics: application to gemcitabine in neuroblastoma-bearing mice. Proc 106th Annu Meet AACR 2015. [61] Braakhuis BJ, Ruiz van Haperen VW, Welters MJ, Peters GJ. Scheduledependent therapeutic efficacy of the combination of gemcitabine and cisplatin in head and neck cancer xenografts. Eur J Cancer Oxf Engl 1990 1995;31A:233540.

[62] Braakhuis BJ, Ruiz van Haperen VW, Boven E, Veerman G, Peters GJ. Schedule-dependent antitumor effect of gemcitabine in in vivo model system. Semin Oncol 1995;22:42-6.

[63] André F, Ciccolini J, Spano JP, Penault-Llorca F, Mounier N, Freyer G, Blay JY, Milano G. Personalized medicine in oncology: where have we come from and where are we going? Pharmacogenomics. 2013;14:931-9.

[64] Yang CG, Ciccolini J, Blesius A, Dahan L, Bagarry-Liegey D, Brunet C, Varoquaux A, Frances N, Marouani H, Giovanni A, Ferri-Dessens RM, Chefrour M, Favre R, Duffaud F, Seitz JF, Zanaret M, Lacarelle B, Mercier C. DPD-based adaptive dosing of 5-FU in patients with head and neck cancer: impact on treatment efficacy and toxicity. Cancer Chemother Pharmacol. 2011;67:49-56.

[65] Ternant D, Cartron G, Hénin E, Tod M, Girard P, Paintaud G. Model-based design of rituximab dosage optimization in follicular non-Hodgkin's lymphoma. Br J Clin Pharmacol. 2012 Apr;73(4):597-605.

[66] Yu KH, Ricigliano M, Hidalgo M, Abou-Alfa GK, Lowery MA, Saltz LB, Crotty JF, Gary K, Cooper B, Lapidus R, Sadowska M, O'Reilly EM. Pharmacogenomic 
modeling of circulating tumor and invasive cells for prediction of chemotherapy response and resistance in pancreatic cancer. Clin Cancer Res. 2014 20(20):5281-9.

[67] Hoskins JM, Goldberg RM, Qu P, Ibrahim JG, McLeod HL. UGT1A1*28 genotype and irinotecan-induced neutropenia: dose matters. J Natl Cancer Inst. 2007;99:1290-5.

[68] Serdjebi C, Seitz JF, Ciccolini J, Duluc M, Norguet E, Fina F, Lacarelle B, Ouafik L, Dahan L. Rapid deaminator status is associated with poor clinical outcome in pancreatic cancer patients treated with a gemcitabine-based regimen.

Pharmacogenomics. 2013;14:1047-51 


\section{TUMOR} MODEL

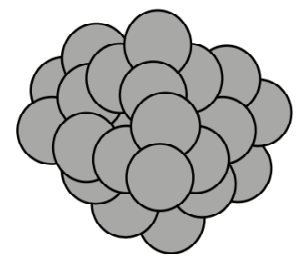

Homogenous cancer cell population

with exponential growth

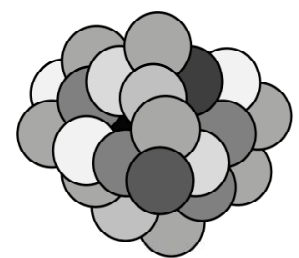

Cancer cells in various phases of the cell

cycle and decreasing growth rate

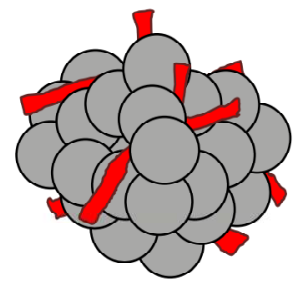

Metronomic chemotherapy concept
Skipper-Schabel-Wilcox

"log-kill" hypothesis

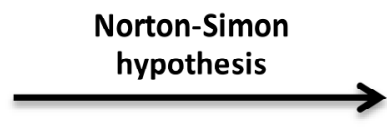

OPTIMAL

SOLUTION

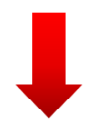

Spaced-out high dose chemotherapy

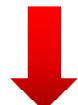

\section{py}

\author{
Spaced-outhigh dosechemotherapy
}



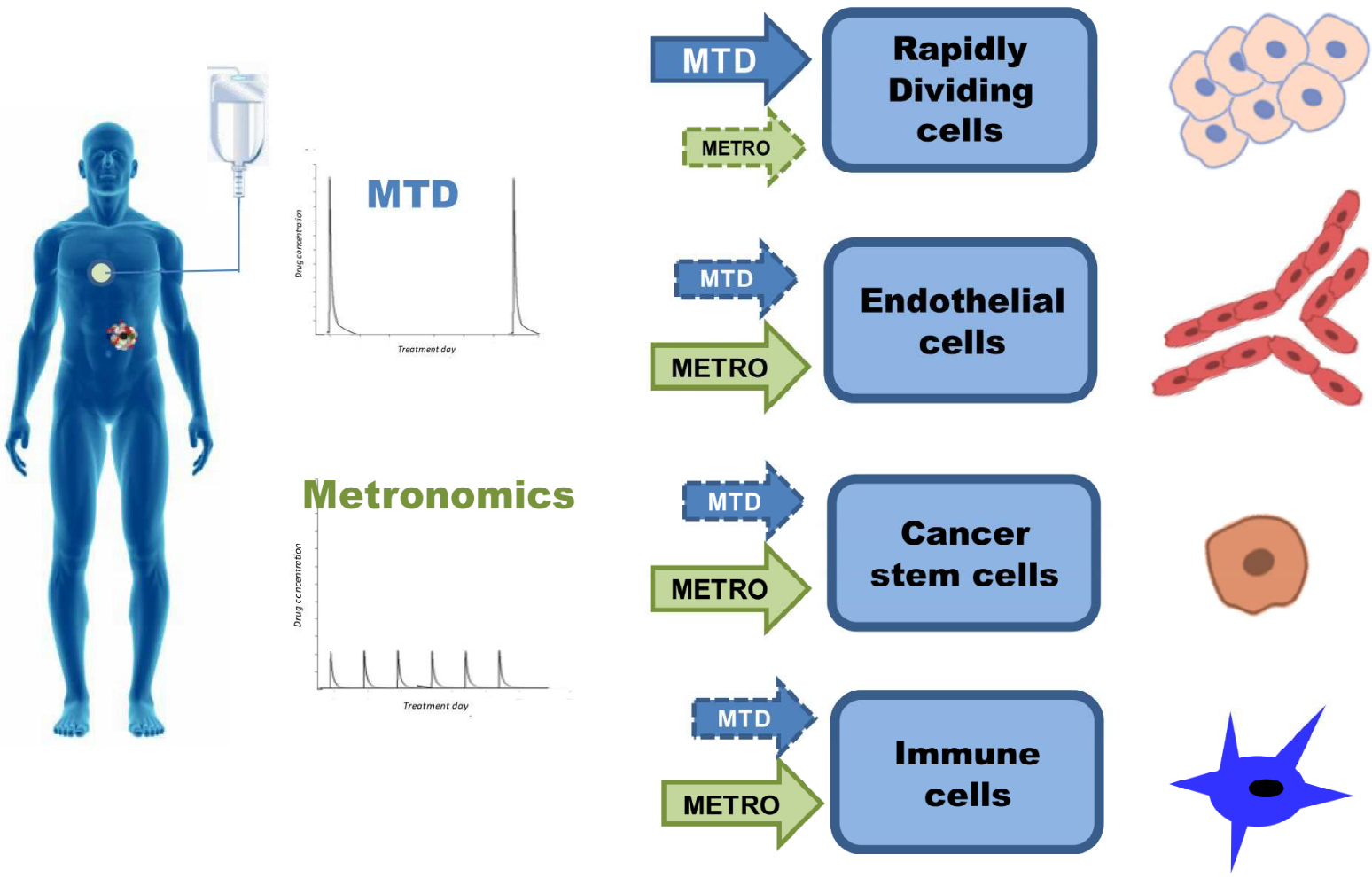
Figure-3
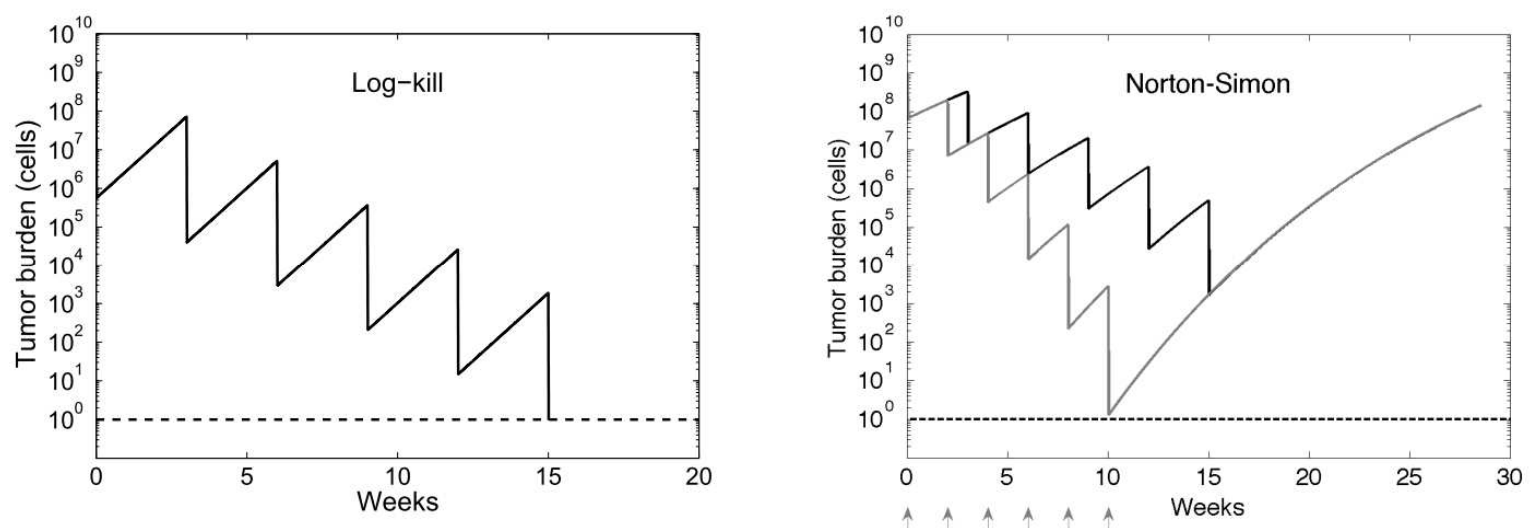


\section{Figure-4}
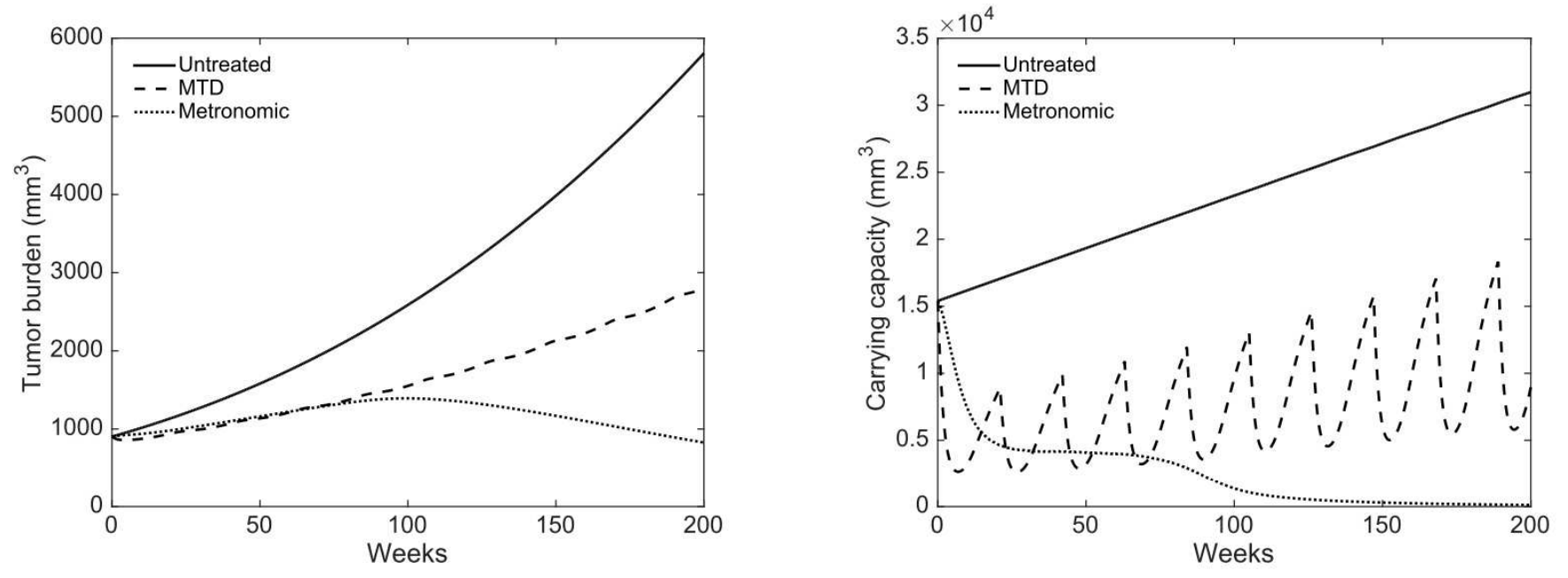

Weeks 
Tumor distinct characteristics
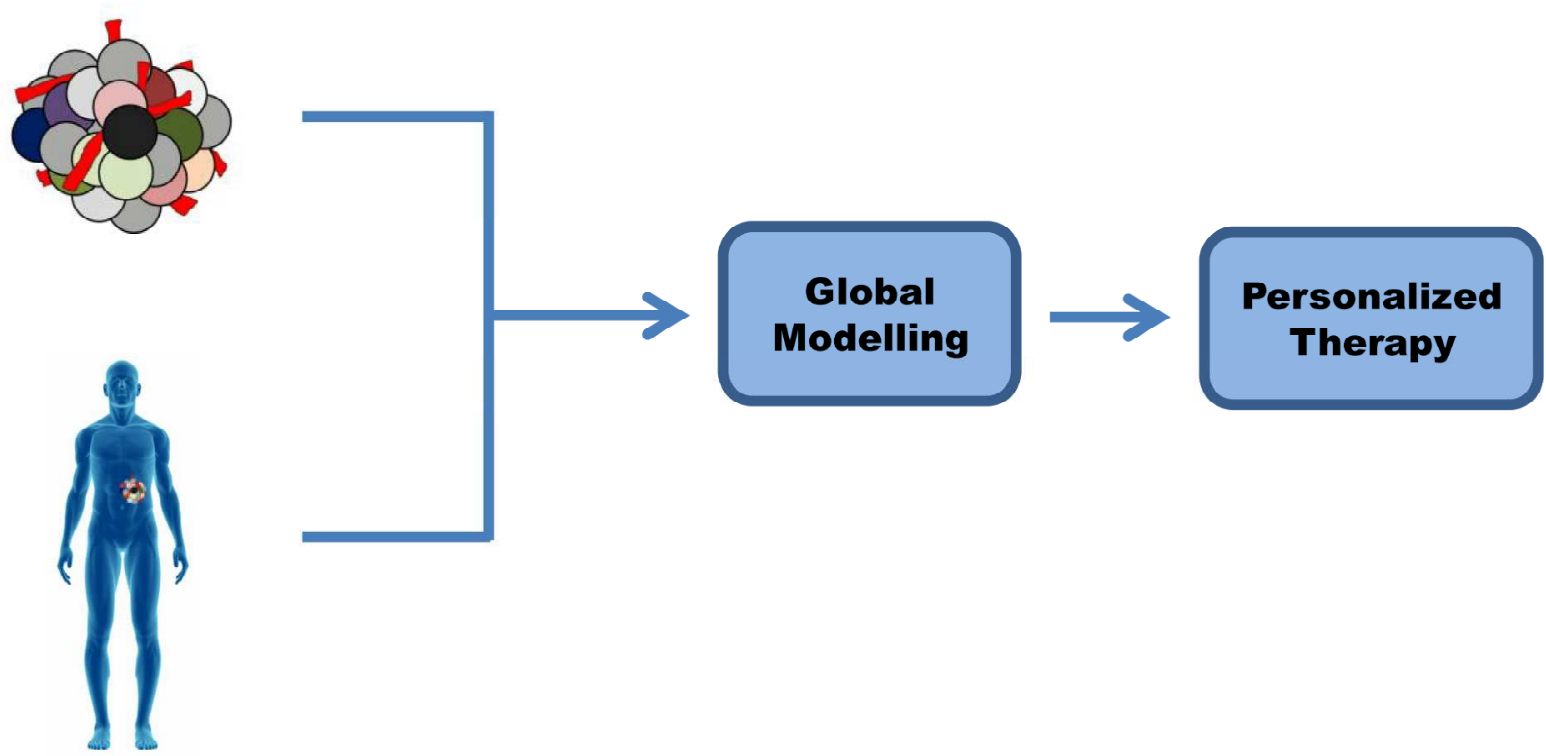

Host distinct characteristics 